\title{
Present Situation and Analysis of Yi Nationality Wrestling in Shilin County
}

\author{
Qiang Yan ${ }^{1, a}$, Dong Huang ${ }^{2}$ \\ ${ }^{1,2}$ College of Physical Education, Yulin University, 719000
}

\begin{abstract}
Wrestling has a long history, and wrestling activities are deeply loved and respected by the people of Shilin which is known as the "Hometown of Wrestling." With the continuous development and progress of society, Yi Nationality's wrestling is facing new opportunities as well as new challenges. Through the methods of literature, data statistics, questionnaire, expert interview and logical analysis, this paper studies 50 long-term wrestlers in Shilin County, analyzes the present situation and finds out the existing problems, thus providing the necessary reference for the development of the Yi wrestling sport.
\end{abstract}

\section{Preface}

Wrestling has a long history, and wrestling activities are deeply loved and respected by the people of Shilin which is known as the "Hometown of Wrestling." In 2011, the Shilin Yi nationality' s wrestling officially became a national intangible cultural heritage. It created a good development space and inheritance environment for the Shilin nationality' s wrestling traditional sports project in Shilin, which made this traditional national sports get better development. In 2015, under the participation and supervision of the leadership of the County Sports Bureau, the Civil Affairs Bureau and the People Sect Bureau, the National Traditional Wrestling Association of Shilin Yi Autonomous County, with the aim of "carrying forward the glamour culture of $\mathrm{Yi}$ Township and inheriting the national traditional wrestling", was founded in the Sani cultural city of Mount Shilin Guihan. In this context, the development of the wrestling of the $\mathrm{Yi}$ in Shilin is particularly important. It will play a positive role in the inheritance and development of the wrestling cause of the Yi in Shilin.

\section{Research purposes and research significance}

\subsection{Formatting the title, authors and affiliations}

With the constant changes of society, modern sports continue to impact the development of traditional sports. How to inherit and protect these minority traditional sports culture is an urgent problem to be solved. Through the historical trace of the culture of the Yi wrestling, the present situation of the development of wrestling culture and the cultural connotation of the wrestling activities,

\footnotetext{
a Corresponding author: author@e-mail.org
}

and combining the local actual situation and drawing on the development experience of other ethnic minority traditional sports, the factors that restrict the development of the wrestling sport of the Yi nationality are found. This will provide advice and suggestions for the sustainable development of the Yi wrestling sport.

\section{Research Objects and Methods}

\subsection{Research objects}

A random selection of 50 enthusiasts who participate in the $\mathrm{Yi}$ nationality wrestling sport in Shilin County, Yunnan Province, is selected as the research object.

\subsection{Research objects}

Research methods

\subsubsection{Literature method}

According to the research content and research purposes of this paper, the Chinese journal full-text database, network database resources, and related literatures such as "Yi wrestling" and "minority traditional sports" are collected to provide a certain knowledge base for field investigation, expert interviews and writing papers.

\subsubsection{Questionnaire method}

By referring to the literature, 50 "questionnaires of Shilin Yi wrestling sports" are designed, distributed and recycled by reasonable methods of sports research. 


\subsubsection{Questionnaire method}

The data obtained from the investigation of the development of wrestling in Shilin are compiled with Excel, and the results of the analysis are obtained, and the related data charts are made to provide the basis for the study.

\section{Results and analysis}

\subsection{The development of the $\mathrm{Yi}$ nationality wrestling in Shilin}

\subsubsection{The time distribution of the wrestling of the Yi nationality in Shilin}

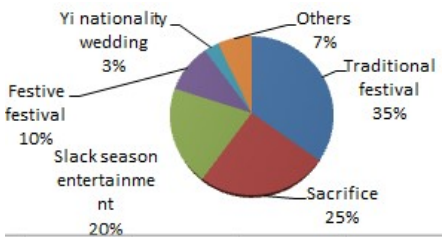

Figure 3-1 The time distribution of the wrestling of the Yi nationality in Shilin

As can be seen from Figure 3-1, traditional festivals, sacrifices, Slack season entertainment and festive festivals are the main time for the Shilin's Yi to wrestle. Through the investigation of the time of the wrestling of the $\mathrm{Yi}$ in Shilin, it is found that the various forms of the wrestling activities of the Yi in Shilin have made the sport of the Yi wrestling better.

\subsubsection{The age of the Yi wrestling participants in Shilin}

Every sport has a suitable age for participation. By investigating the age groups of the $\mathrm{Yi}^{\prime} \mathrm{s}$ wrestling sport, the appropriate age of the $\mathrm{Yi}$ wrestling project can be reflected, and the mass base of the sport can also be reflected.

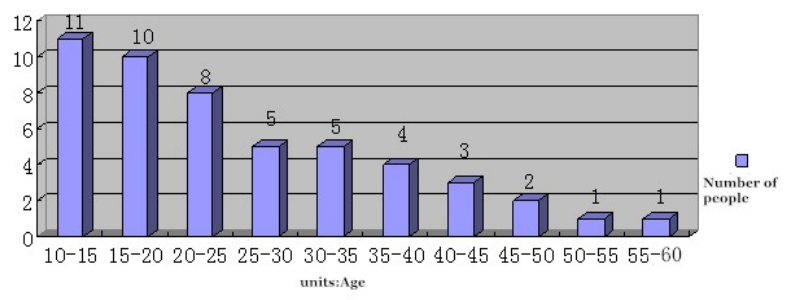

Figure 3-2 The age of the Yi wrestling participants in Shilin

It can be seen from Figure 3-2 that the age of the participants of the Yi wrestling is mainly between 10-15 years old and 15-20 years old, and the number of people in the two age groups is 11 and 10 respectively; The second group is 8 people aged 20 to 25 , indicating that the age group of participants in the Yi wrestling is mainly teenagers. This is closely related to the prevalence of the local wrestling and children are taught by their parents from an early age, and have a strong interest in wrestling.
At the same time, it can be seen from the data that the age of the participants in the wrestling of the Shilin is very scattered, with the participation of teenagers and young children as well as the participation of the middle-aged and the elderly, which shows that the wrestling in Shilin has a thick mass foundation.

\subsubsection{Gender status of participants in the Shilin Yi's wrestling}

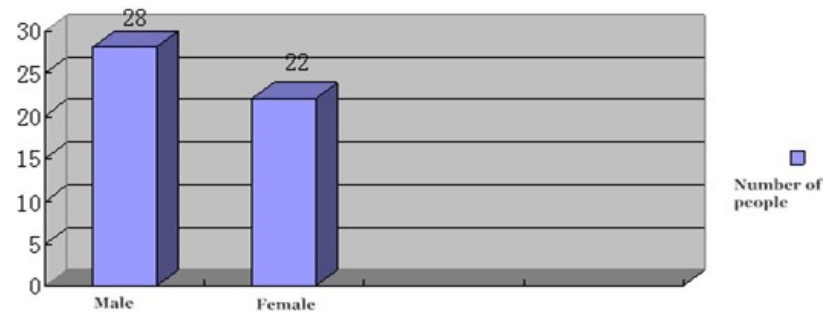

Figure 3-3 Gender statistics of participants in the Shilin Yi' s wrestling

As can be seen from Figure 3-3, women who take part in wrestling account for $44 \%$, while men account for $56 \%$. This also reflects the participation of the $\mathrm{Yi}$ in wrestling activities regardless of men and women, but also reflects the love and participation of the Yi's men and women in wrestling activities.

\subsubsection{Investigation on motivation of participants in Shilin Yi wrestling}

Psychology holds that "human behavior has certain motives, and motivation arises from the inherent needs of mankind". People's behavior is generally purposeful, and it is a process of achieving a certain goal driven by some kind of motivation.

Table 3-2 Analysis of the investigation of the motivation of the participants of the Shilin Yi ' $\mathrm{s}$ wrestling $(\mathrm{N}=50)$

\begin{tabular}{lcc}
\hline Motivation & Number of people & Percentage \\
\hline Following customs and habits & 16 & $32 \%$ \\
Inheriting the culture of the Yi Nationality & 14 & $28 \%$ \\
Building up a good physique and improve health & 6 & $12 \%$ \\
Exercising will & 5 & $10 \%$ \\
Enriching the leisure life & 4 & $8 \%$ \\
Interpersonal communication & 1 & $2 \%$ \\
Others & 4 & $8 \%$ \\
\hline
\end{tabular}

By investigating the motivation of $\mathrm{Yi}$ wrestling athletes, the motivation for participating in the wrestling of the Yi nationality is: Following customs and habits, inheriting the culture of the Yi Nationality, building up a good physique and improve health, exercising will, enriching the leisure life, interpersonal communication and others. The proportion is $32 \%, 28 \%, 12 \%, 10 \%, 8 \%$, $2 \%$ and $8 \%$ respectively.

Through the analysis of the data of Table 3-2, it can be seen that the biggest motivation of the participants in the wrestling of the Yi ethnic group is to follow the customs and habits, and the second is to inherit the Yi culture, build up a good physique and improve health, exercise will and so on. It reflects the diversity of motivations of participants in the Shilin Yi wrestling and promotes the 
continuous development of the Yi wrestling sport.

\subsubsection{Number of times of participation of participants in Yi's wrestling activities each year}

The popularity and prevalence of the sport can be seen based on the number of times the participant participates in the event each year.

Table 3-3 Analysis of the number of times of participation of participants in the Yi's wrestling activities each year $(\mathrm{N}=50)$

\begin{tabular}{lcc}
\hline Education & Number of people & Percentage \\
\hline $1-2$ times & 8 & $16 \%$ \\
$3-4$ times & 15 & $30 \%$ \\
$5-6$ times & 17 & $34 \%$ \\
6 times and above & 10 & $20 \%$ \\
\hline
\end{tabular}

Through table 3-3, it can be seen that the number of athletes participating in the Yi wrestling is $16 \%$ in $1-2$ times, $30 \%$ in $3-4,34 \%$ in $5-6$, and $20 \%$ in more than 6 times. It reflects that the $\mathrm{Yi}$ people are actively participating in the wrestling, and the Yi wrestling is popular with the Yi people of the Shilin, and it also reflects the broad masses foundation of the wrestling movement of the Yi nationality in Shilin.

\subsection{Factors affecting the development of the Yi wrestling in Shilin}

\subsubsection{The impact of modern sports}

With the continuous development of modernization, many sports such as basketball, football, badminton, and table tennis have been introduced into the Yi area, and they have gained popularity and development. Minority people have more choices for sports activities. Therefore, the Yi' s wrestling activities are facing certain impacts and challenges.

\subsubsection{The shrinking of wrestling matches}

In the survey and interviews, the activities of the $\mathrm{Yi}$ wrestling have been reduced. Compared with the past, the Yi's wrestling activities have gradually decreased in recent years. In some traditional ritual activities and festive festivals of the Yi nationality, even the $\mathrm{Yi}^{\prime} \mathrm{s}$ wrestling activities have not been carried out, which is not conducive to the inheritance and development of the $\mathrm{Yi}^{\prime} \mathrm{s}$ wrestling, and is not conducive to the participation of wrestling enthusiasts.

\subsubsection{The impact of modern media technology}

With the development of society, the continuous improvement of infrastructure and the development of modern media technology, it has changed the life concept and lifestyle of the area of Yi nationality, and gradually increased the communication and contact methods between the formerly backward and backward Yi nationality and the outside world. Many modern recreational activities flow into the Yi nationality area along with the development of information technology. These recreational activities continue to enrich the life and spiritual world of the Yi people, and to some extent affect the spread of the traditional sports of the Yi people, and have influenced the inheritance and development of the Yi traditional sports to a certain extent.

\subsubsection{The lack of knowing and understanding of wrestling culture}

In the traditional wrestling competition, participation in wrestling is unpaid, in order to follow customs and honors and so on, which embodies the diligent and brave spirit of the Yi people. The prizes of the winners are generally a few red cloths. After winning the game on the court, the contestants will walk around the circle for a few laps. After returning to the village, they will be the pride of the people of the whole village. They are the heroes in everyone's mind. With the development of social economy and the process of urbanization, the living conditions of the Yi have been improved. More and more people go out of the mountain and go to the city, gradually reducing the participation of wrestling movement and knowing and understanding of the wrestling culture, which has influenced the inheritance and development of the traditional wrestling of the $\mathrm{Yi}$ nationality to a certain extent.

\section{Conclusions and suggestions}

\subsection{Conclusions}

\subsubsection{High participation of the masses}

Wrestling is one of the favorite and popular traditional sports of the Yi nationality in Shilin. The living area of Shilin Yi's people will organize their own wrestling activities, no Yi's men can't wrestle, no Yi's women don't like wrestling. In the wrestling field, the children of the age of ten to as old as 50 to 60 will participate in the wrestling sport. Therefore, the Yi's wrestling has a high participation and a solid mass base.

\subsubsection{The carrying of traditional festival activities}

The wrestling activities of the Yi nationality in Shilin are mainly carried out on traditional festivals or festive festivals. Wrestling is an essential part of the traditional festivals such as the "Spring Festival", "Dragon Boat Festival", "Mid-Autumn Festival", "Torch Festival", "Secret Branch Festival" and "Dragon Festival". The Yi ' $\mathrm{s}$ wrestling activities carried by traditional festivals have continuously injected new vitality into the Yi's wrestling, which makes the Yi's wrestling inheritance more stable and passed down from generation to generation. 


\subsubsection{Promotion and support from government departments}

The development of the Yi's wrestling is inseparable from the promotion and support of the government departments. The government departments actively protect and develop the Shilin Yi's wrestling sport and its wrestling culture, and continue to give support in policy. At the same time, they have made great support in holding wrestling competitions, funds, venues, etc., which has brought about continuous development of the Shilin $\mathrm{Yi}^{\prime} \mathrm{s}$ wrestling sport, and the $\mathrm{Yi}^{\prime} \mathrm{s}$ wrestling sport has been better developed.

\subsubsection{Mass organization and enterprise support}

In each village where the Yi people live in Shilin, they will organize their own wrestling activities, which will be passed down from generation to generation through competitions and continue to this day. In the process of modern social development, some local enterprises will also vigorously support the development of wrestling activities. More fresh blood has been injected into the development of $\mathrm{Yi}^{\prime} \mathrm{s}$ wrestling by means of funds and propaganda.

\subsection{Suggestions}

\subsubsection{Further strengthening people's understanding of the Yi's wrestling culture in Shilin}

The Yi nationality wrestling culture is an indispensable part of the Yi culture. Through verbal narratives, wrestling activities, writing books on Yi' s wrestling, and promoting the culture of $\mathrm{Yi}^{\prime} \mathrm{s}$ wrestling, the $\mathrm{Yi}$ people can have a clear knowing and understanding of the cultural values, educational values and economic values behind the traditional wrestling activities of the Yi. While inheriting and protecting the Yi wrestling culture, more people are actively involved.

\subsubsection{Combining school sports with traditional national sports}

The combination of Yi wrestling and school sports should be carried out to carry out local characteristic courses. The development of the Yi wrestling movement in school sports can exercise the students' body, promote the healthy and happy growth of the students, form a good psychological quality, promote the all-round development of the students' personality, and cultivate the habit and consciousness of the students' lifelong physical exercise. Wrestling can also enable students to fully understand the $\mathrm{Yi}^{\prime} \mathrm{s}$ wrestling culture and promote the development of Yi wrestling.
With the continuous development of society, the $\mathrm{Yi}^{\prime} \mathrm{s}$ wrestling should speed up reform and innovation, extract the essence and remove the dross. According to the local conditions of Shilin County and the current situation of the development of $\mathrm{Yi}^{\prime} \mathrm{s}$ wrestling, we should proceed from the reality and correctly handle the relationship between inheritance and development, learning and innovation. We must conscientiously summarize the experience of the development of the Shilin $\mathrm{Yi}^{\prime} \mathrm{s}$ wrestling, and strengthen exchanges with other outstanding national traditional sports programs, learn and draw on the advanced concepts and experiences suitable for the development of the Yi' s wrestling, so that the $\mathrm{Yi}^{\prime} \mathrm{s}$ wrestling activities can be better passed down and developed.

\subsubsection{Combining the Shilin Yi wrestling with international wrestling}

The rules of the Shilin Yi' s wrestling are very similar to the rules of international freestyle wrestling. In the course of the development of Shilin Yi wrestling, many Shilin Yi's wrestlers, under the influence of the Yi's wrestling culture at a young age, have been participating in the traditional folk wrestling activities in Shilin area continuously, which has laid a solid foundation for them to go to the international arena and achieved excellent results in the domestic and foreign competitions. Therefore, it is more conducive to the vigorous development of Yi's wrestling to connect traditional Yi's wrestling with international wrestling.

\section{References}

1. Liu Yang. The Evolution and Inheritance of the Yi' s Wrestling in the Process of Urbanization [D]. Chengdu Sport University, 2015: 10-12.

2. Li Xiaosong. Investigation and Prospect of Yunnan Minority Wrestling Activities [D]. Chengdu Sport University, 2015: 21-25.

3. Zhang Ping. The Sani Wrestling of Yunnan Stone Forest Yi Special Cultural Symbol [D]. Yunnan Minzu University, 2014: 34-38.

4. $\mathrm{Wu}$ Zhaoming. Study on the Formation, Evolution and Development Countermeasures of the Traditional Wrestling Activities of the Yi Nationality in Yunnan [D]. Yunnan Normal University, 2005: 24-26.

5. Chen Yonghui, Bai Jinxiang. Protection of Chinese Minority Folk Sports Culture from the Perspective of Immaterial Cultural Heritage [J]. Journal of Physical Education, 2009, (05): 91-94.

6. Fan Liling. From Traditional Games to Performance - The Change of Shilin Yi Wrestling Culture [J]. Sports World (Scholarly), 2009, (02): 64-66.
4.2.3 Handling the relationship between $\mathrm{Yi}^{\prime} s$ wrestling inheritance and development, learning and innovation 